\title{
Microcystic Adnexal Carcinoma Misdiagnosed as Desmoplastic Trichoepithelioma on Preoperative Biopsy
}

\author{
Sung Hoon Koh, \\ Kwang Rae Kang, \\ Ji Hoon Yang, \\ Sung Won Jung, \\ Hyuck Jae Lee
}

Department of Plastic and

Reconstructive Surgery, Hallym University

Sacred Heart Hospital, Hallym University

Medical Center, Anyang, Korea

No potential conflict of interest relevant to this article was reported.

\begin{abstract}
Microcystic adnexal carcinoma is a rare type of tumor, with about 300 cases reported globally. Due to its similar histology with other tumors, it is occasionally misdiagnosed as desmoplastic trichoepithelioma, basal cell carcinoma, syringoma, and so on. We present a patient with a mass on the perioral area who was preoperatively diagnosed with trichoepithelioma. Microcystic adnexal carcinoma was diagnosed after excisional biopsy and a wide excision. Defects were reconstructed with a mucosal advancement flap. There was no recurrence and there were no significant complications during the 18-month follow-up period. Because superficial punch biopsy has limitations in width and depth, surgeons should always consider the possibility of malignancy of a mass even if a biopsy shows a benign result.
\end{abstract}

Keywords: Skin cancer / Trichoepithelioma / Misdiagnosis

\section{INTRODUCTION}

Microcystic adnexal carcinoma (MAC) was first described as a slowly growing but locally aggressive adnexal neoplasm in 1982 [1]. MAC is an uncommon type of tumor, with about 300 cases reported globally. MAC occurs in functionally and cosmetically sensitive anatomic locations, mostly on the face, so distinguishing between benign and malignant lesions for accurate diagnosis is important for proper patient management planning [2-6]. However, MAC is occasionally misdiagnosed as other benign or malignant neoplasms, such as desmoplastic trichoepithelioma, basal cell carcinoma, squamous cell carcinoma, and syringoma due to their histological similarity $[3,4,7]$. Here, we present a case of MAC misdiagnosed as desmoplastic trichoepithelioma on preoperative biopsy.

Correspondence: Sung Won Jung

Department of Plastic and Reconstructive Surgery, Hallym University Sacred Heart Hospital, Hallym University Medical Center, 22 Gwanpyeong-ro, 170 beon-gil, Dongan-gu, Anyang 431-796, Korea

E-mail: hallymjsw@gmail.com

Received September 18, 2014 / Revised November 20, 2014 / Accepted April 2, 2015

\section{CASE REPORT}

A 66-year-old woman with a mass on her face was transferred to us from dermatology for excision. There were multiple skin-colored indurate nodules in the right perioral area (Fig. 1). She had first noticed it 20 years previously. At that time, it was a small pimple-like mass. It had grown very slowly for 20 years since then. On palpation, the mass was firm, immovable, and located at the subcutaneous level.

The lesion was diagnosed as desmoplastic trichoepithelioma in a preoperative punch biopsy carried out by a dermatologist (Fig. 2A). While immunohistochemistry can help distinguish MAC from other tumors, it was not done by our pathology department.

We performed an excisional biopsy of the lesion under local anesthesia. Contrary to our expectation, intraoperative findings showed that the lesion was ill-defined and invading the perioral muscles and vessels. We could not perform a complete excision due to the tumor's ill-defined margins and benign preoperative biopsy diagnosis. However, the postoperative biopsy showed that tumor cells had invaded the muscle layer and also showed perineural 
invasion, so the result was MAC (Fig. 2B, C).

Based on these findings, we planned to make a wide excision under general anesthesia 12 days after the first operation. The incision was made along the vermilion border with margins of $2 \mathrm{~mm}$ from the previous incision line (Fig. 3). We resected not only the tumor but also the adjacent muscle, vessels, overlying lip, perioral

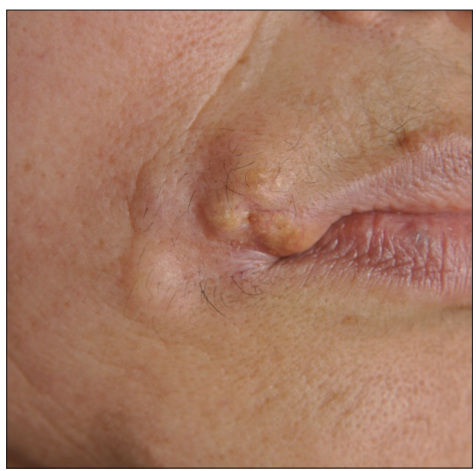

Fig. 1. A 66-year-old woman presented with a slowly but progressively growing mass in the right perioral area. The mass was firm, immovable and consisted of multiple subcutaneous nodules. skin and oral mucosa (Fig. 4). Resected tissues were sent for frozen biopsy, and there was remnant MAC tissue on the lateral margin. Therefore, we carried out another further resection of $2 \mathrm{~mm}$ from the lateral margin. The excised muscles were repaired, and defect of the lip was reconstructed by mucosal advancement flap.

During the first 18 months after surgery, there was no significant complication or local recurrence. Although motion of the mouth was slightly restricted, the patient was satisfied with the results (Fig. 5).

\section{DISCUSSION}

MAC is an uncommon type of tumor. Generally, MAC can present variable features from a smooth flesh-colored or yellow plaque to a cystic nodule, as seen in this case. It was difficult to suspect MAC with only the clinical presentation of our patient, even with the result of desmoplastic trichoepithelioma on preoperative bi-
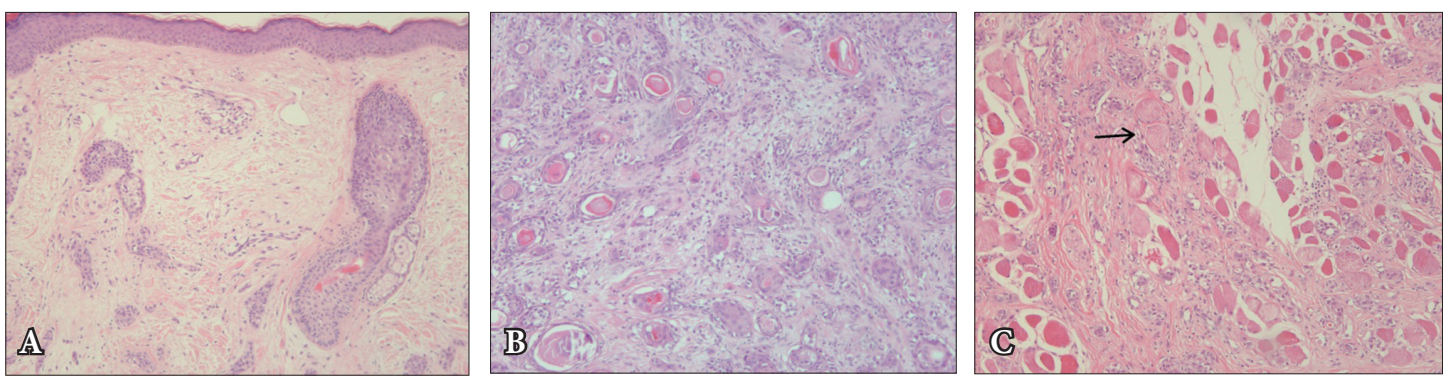

Fig. 2. (A) Histopathologic image of the preoperative superficial biopsy specimen (H\&E, $\times 100)$. Well-confined and symmetrical proliferations of narrow strands of basaloid epithelium form small nests and cords. There is no keratin cyst that is usually seen in desmoplastic trichoepithelioma. $(\mathrm{B}, \mathrm{C})$ Histopathologic images of the postoperative biopsy specimen $(\mathrm{H} \& \mathrm{E}, \times 100)$. There are keratin cysts, small nests and cords of basaloid cells also seen in the preoperative biopsy. However, the tumor cells have invaded the muscle layer, and perineural invasion is also seen (arrow).
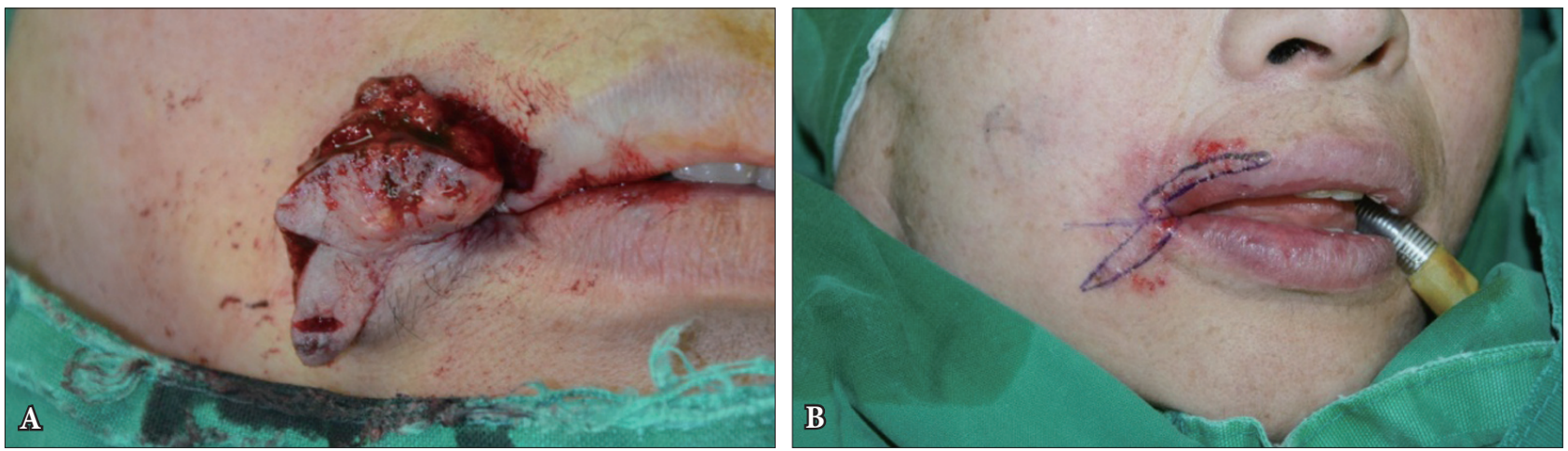

Fig. 3. (A) Intraoperative photograph showing the excised mass in the first operation. An incision was designed along the margin of the nodules. (B) Preoperative photograph showing a resection margin of $2 \mathrm{~mm}$ from the previous incision line in the second operation. 

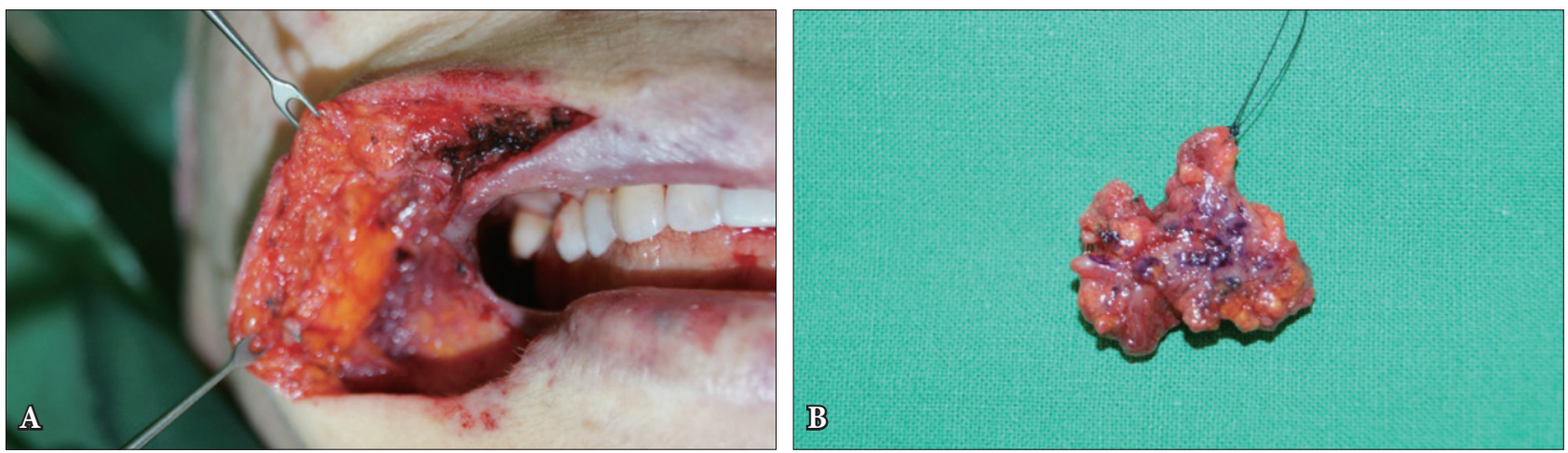

Fig. 4. Intraoperative photographs of (A) patient and (B) excised tumor. The tumor was excised widely including surrounding skin, muscle, vessels, and oral mucosa. The ill-defined mass had invaded the perioral muscles and vessels.
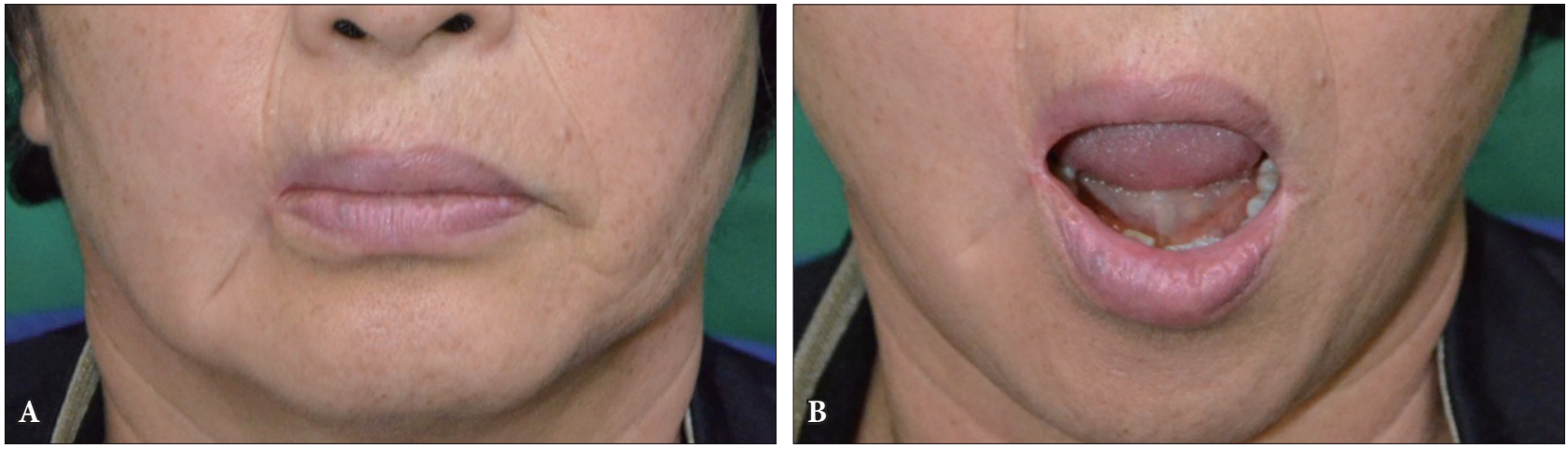

Fig. 5. (A, B) Photographs at 18-month follow-up visit. The patient was satisfied with mouth opening and lip motion.

opsy. However, because the feature of our patient-multiple nodules that grew for 20 years-is not commonly presented, the possibility of malignancy should be considered.

MAC rarely leads to death [8]. However, because it is malignant, complete resection with clear surgical margins must be done to prevent recurrence. Although, there is currently no clearly suggested resection margin for treatment. Multiple case series and one prospective study proved that treatment with Mohs micrographic surgery (MMS) results in a low recurrence rate [9]. Abbate et al. [10] reported that an MMS group showed a lower recurrence rate than a wide excision group. These results suggest that MMS is a reasonable first-line therapeutic modality for MAC [2].

There is no consensus regarding the follow-up period for MAC, but the recurrence rates for conventional excision may be as high as $47 \%$, usually within the first 3 years. For MMS, the recurrence rates ranges from $0 \%$ to $22 \%$ with a 5 -year follow-up $[4,9]$. The indolent course of the tumor is well described, and re- currences have been described 30 years after initial treatment. Therefore, irrespective of treatment modality, all patients should be monitored closely for several decades [10,11].

MACs are mostly located in the perioral and paranasal areas, which are functionally and cosmetically sensitive locations. Defects and deformities on the face resulting from wide excision are inevitable, despite the reconstructive efforts of surgeons. Clearly, accurate diagnosis and appropriate treatment are very important.

Superficial punch biopsy is a time and cost-effective procedure. However, it has led to a decrease in the size of tissue samples submitted for histopathologic examination. Also, it occasionally leads to difficulty in differential diagnosis because the pathologist cannot see the exact breadth or depth of the tumor. In one case study, misdiagnosis occurred in 27\% of MAC cases, and that is secondary to inadequate sampling [2]. This is a limitation of superficial punch biopsy, and occasional misdiagnosis is inevitable. MAC shows pathologic findings that are similar to those of desmoplas- 
tic trichoepithelioma, and the depth is a critical clue for diagnosis.

Surgeons should be aware of the possibility of misdiagnosis and get informed consent from patients as the diagnostic change from benign to malignant is upsetting for them. In cases like MAC, surgeons should sufficiently inform and explain to patients for them to understand that their diagnosis may change depending on biopsy results.

\section{REFERENCES}

1. Goldstein DJ, Barr RJ, Santa Cruz DJ. Microcystic adnexal carcinoma: a distinct clinicopathologic entity. Cancer 1982;50:566-72.

2. Chiller K, Passaro D, Scheuller M, Singer M, McCalmont T, Grekin RC. Microcystic adnexal carcinoma: forty-eight cases, their treatment, and their outcome. Arch Dermatol 2000;136:1355-9.

3. Ohtsuka H, Nagamatsu S. Microcystic adnexal carcinoma: review of 51 Japanese patients. Dermatology 2002;204:190-3.

4. Friedman PM, Friedman RH, Jiang SB, Nouri K, Amonette R, Robins
P. Microcystic adnexal carcinoma: collaborative series review and update. J Am Acad Dermatol 1999;41:225-31.

5. Choi YW, Oh SJ, Lee JW, Jang YC, Chae SW, Sohn JH. A clinical experience of microcystic adnexal carcinoma in lower lip and back. J Korean Soc Plast Reconstr Surg 2002;29:341-4.

6. Kim IJ, Lim JS, Ahn ST, Lim P. A case of microcystic adnexal carcinoma. J Korean Soc Plast Reconstr Surg 1993;20:844-8.

7. Billingsley EM, Fedok F, Maloney ME. Microcystic adnexal carcinoma. Case report and review of the literature. Arch Otolaryngol Head Neck Surg 1996;122:179-82.

8. Yugueros P, Kane WJ, Goellner JR. Sweat gland carcinoma: a clinicopathologic analysis of an expanded series in a single institution. Plast Reconstr Surg 1998;102:705-10.

9. Diamantis SA, Marks VJ. Mohs micrographic surgery in the treatment of microcystic adnexal carcinoma. Dermatol Clin 2011;29:185-90.

10. Abbate M, Zeitouni NC, Seyler M, Hicks W, Loree T, Cheney RT. Clinical course, risk factors, and treatment of microcystic adnexal carcinoma: a short series report. Dermatol Surg 2003;29:1035-8.

11. Lupton GP, McMarlin SL. Microcystic adnexal carcinoma. Report of a case with 30-year follow-up. Arch Dermatol 1986;122:286-9. 